Rita Brdar-Szabó

Budapest

\title{
Bildschemata bei Resultativkonstruktionen im Deutschen und Ungarischen
}

\author{
DOI: 10.14232/fest.bassola.1
}

\begin{abstract}
Der vorliegende Beitrag widmet sich der vergleichenden Untersuchung von Resultativkonstruktionen im Deutschen und Ungarischen. Vordergründiges Ziel der Studie ist die kontrastive Analyse der motivierenden Rolle von Bildschemata, wobei interlinguale Unterschiede und Ähnlichkeiten in ihren Erscheinungsformen herausgestellt werden. Die Ergebnisse der kontrastiven Analyse werden in einer tabellarischen Übersicht präsentiert. Die Arbeit versteht sich einerseits als ein erster bescheidener Baustein zu einer kognitiv-linguistischen Typologie von Resultativkonstruktionen, andererseits bietet sie Material für praxisbezogene Anwendungen im DaF-Unterricht und in der Translatologie.
\end{abstract}

\section{Einleitung und Problemstellung}

Untersuchungsgegenstand des vorliegenden Beitrags sind Resultativkonstruktionen im Sprachvergleich. Diese können hier jedoch aus Umfangsgründen nicht umfassend behandelt werden, zumal die einschlägige Forschungsliteratur im übereinzelsprachlichen Maßstab vor allem im Anschluss an die Veröffentlichung von Goldberg / Jackendoff (2004) beinahe unüberblickbar geworden ist. Diese Feststellung bezieht sich nicht nur auf das Englische, sondern auch auf das Deutsche, ${ }^{1}$ wo die Resultativkonstruktion beinahe als eine Modeerscheinung ${ }^{2}$

1 Das Ungarische stellt diesbezüglich eine Ausnahme dar, zumal es hier meines Wissens erst zwei aktuell laufende Projekte gibt, Brdar-Szabó (2019) speziell zu Resultativkonstruktionen und Kugler (2019) zu den Verben der Zustandsveränderung im Allgemeinen.

2 Ágel (2017: 424) spricht hier von „dem konstruktionsgrammatischen Resultativ-Boom.“ 
angesehen werden kann. Ágel (2017: 423f.) stellt mit Recht fest, dass "Resultativkonstruktionen in der modernen Grammatikforschung im Zentrum des Interesses [stehen], da sie für jede Grammatiktheorie eine besondere Herausforderung darstellen." Ihre Problematik soll hier aus der Perspektive der Bildschemata im Rahmen einer kognitiv-linguistisch begründeten kontrastiven Linguistik angegangen werden, Vergleichssprachen sind das Deutsche und das Ungarische.

Im Folgenden soll zuerst das hier interessierende Phänomen anhand von Beispielen vorgeführt, definiert und abgegrenzt werden.

(1) Die Wolle wird dann zu verschiedenen Produkten verarbeitet, beispielsweise zu einem Pullover oder zu Alpakawolle-Bettdecken.

https://www.dormando.de/lexikon/alpakawolle/ [gesehen am 23.02.2019]

(2) Wir gehen teilweise unter den Felsen hindurch, kommen wieder an die Oberfläche, wo das Wasser die Felsen glatt schmirgelte.

https://www.fotocommunity.de/photo/unter-dem-felsbrocken-schwedenjani/39501622 [gesehen am 23.02.2019]

(3) Hogyan kell felverni a tojásfehérjét kemény habbá? https://www.gyakorikerdesek.hu/etelek-italok__sutes-fozes__178325 [gesehen am 23.02.2019]

(4) Csiszoljuk simára a polcot, majd töröljük át száraz, puha ruhával. http://www.origo.hu/itthon/20020611 kenyelem.html [gesehen am 23.03. 2019]

Ausgangspunkt für meine Überlegungen ist Goldberg (1995: 4), wo Konstruktionen wie folgt definiert werden: , a form-meaning pair $\left\langle\mathrm{F}_{i}, \mathrm{~S}_{\mathrm{i}}>\right.$ such that some aspect of $F_{i}$ or some aspect of $S_{i}$ is not strictly predictable from C's component parts or from other previously established constructions. " Resultativkonstruktionen lassen sich in diesem theoretischen Rahmen in einer ersten Annäherung als „constructions containing a resultative phrase“ (Broccias 2008: 27) definieren, und ihre Funktion wird in der Kodierung einer kausativen Relation zwischen zwei Ereignissen innerhalb eines einzigen Satzes bestimmt (vgl. Broccias 2004: 103). Folgendes Strukturschema gilt für diese Konstruktion im Englischen und für viele andere Sprachen: $\mathrm{NP}_{1} \mathrm{~V} \mathrm{NP}_{2} \mathrm{RP}$ (Resultativphrase). Nach Levin (1993: 101) ist die Resultativphrase (RP) eine solche Phrase, die 
den durch den Referenten von $\mathrm{NP}_{2}$ erreichten Zustand beschreibt, welcher als Ergebnis der durch das Verb bezeichneten Handlung zustande kommt, was mit anderen Worten so viel bedeutet, dass die Subjekt-NP auf die postverbale NP (d.h. das Akkusativobjekt) einwirkt, indem eine durch die RP spezifizierte Änderung in ihrer Form, Gestalt oder Qualität herbeigeführt wird. Helbigs Definition der Resultativkonstruktionen steht damit völlig im Einklang, bezeichnen doch diese nach ihm „den Nachzustand eines Objektsreferenten, der erst als Resultat der durch die vom Verb bezeichneten Handlung verursacht wird (also vorher nicht vorhanden war.)“ (Helbig 2008: 202, Unterstreichungen im Original). Der helbigsche Nachzustand kann sich dabei ebenfalls auf Form, Gestalt oder Qualität der durch das Objektsprädikativ bezeichneten Entität beziehen. In Ágel (2017) wird eine neue semantische Rolle eingeführt, ${ }^{3}$ welche als RESULTATIVPATIENS (HANDLUNGSRESULTAT) bezeichnet und wie folgt definiert wird: „Sie beschreibt ein konstruktionell erzeugtes, dynamisches Patiens, das als Resultat einer vorangehenden Tätigkeit perspektiviert wird." (Ágel 2017: 425). Die Betonung der Perspektivierung eines dynamischen Patiens als Ergebnis einer vorangehenden Handlung halte ich für besonders wichtig, und dies dient mir als Ausgangspunkt für meine weiteren Überlegungen.

\section{Definitionsprobleme aus kontrastiver Sicht}

Während sich im Ungarischen die ausgewählten Resultativkonstruktionen einheitlich verhalten und alle Entsprechungen der deutschen Subtypen mit Ausnahme von Äquivalenten der Konstruktionen mit $z u+$ Dativ am Objektsprädikativ die Endung -ra/-re aufweisen, zeigen die deutschen Resultativkonstruktionen ein gespaltenes Verhalten: Die als adverbiale Adjektive eingeordneten Ergänzungen verfügen über grundlegend andere Eigenschaften als die direktionalen Präposi-

Hier soll auch die Ersterwähnung genau zitiert werden: „Da das Zielszenario ein Zustandsveränderungsszenario ist und daher das Akkusativobjekt das Resultat einer Zustandsveränderung indiziert, nenne ich diese dynamische, da qua Formel 'angewandte' semantische Rolle (als Wert) HANDLUngSResultat." (Vgl. Ágel 2017: 273). 
tionalobjekte. Eisenberg (1989: 225) weist bereits in der ersten Fassung seiner Grammatik darauf hin, dass objektbezogene Adjektive bei ,kausativen' Verben - wie in (5-7) ${ }^{4}$ gezeigt - „zum morphologischen Bestandteil des Verbs“ werden.

(5) Helga streicht ihr Fahrrad grün (Eisenberg 1989: 224)

(6) Ödipus schlägt seinen Vater tot (ebd.)

(7) Karl kocht die Kartoffeln weich (ebd.)

Über solche Beispiele äußert sich Eisenberg (1989: 225) wie folgt:

Für die Beispiele in $19^{5}$ ist nicht zweifelhaft, ob wir Verben wie grünstreichen, totschlagen oder weichkochen ansetzen sollen. Dagegen scheinen etwa krankschreiben und gesundbeten zweifelsfrei eine Einheit zu sein. Das Adjektiv erhält mehr und mehr den Status einer trennbaren Verbpartikel, krankschreiben und gesundbeten sind analog gebaut zu abgeben und aufhängen [...]. (Eisenberg 1989: 225)

Eisenberg scheint in der früheren Version seiner Grammatik Wortbildung, Produktivität und Lexikalisierung nicht strikt voneinander zu trennen, Eisenberg (1998: 257) schafft aber klare Fronten und äußert sich über dieses Problem präziser:

Die Bildung von Verbstämmen mit adjektivischen Partikeln (7c) hat ebenfalls als produktiv zu gelten. Solche Formen entstehen durch Verwendung eines Adjektivs als Objektsprädikativ in einer dem Resultativ verwandten Funktion wie in Er putzt ihre Schuhe blank. Durch Inkorporation des Adjektivs als Verbpartikel entsteht ein neues transitives Verb (etwas blankputzen; jemanden krankschreiben). Die Zahl der Verben dieses Typs ist groß und prinzipiell offen. (Eisenberg 1998: 257)

Eisenberg spricht auf derselben Seite vom „Übergangsbereich zwischen Wort- und Satzgrammatik“ (vgl. Eisenberg 1998: 257). Dass der Autor an dieser Konzeption konsequent festhält, sieht man daran, dass er die einschlägigen Passagen in allen

\footnotetext{
Bei Eisenberg sind die Beispiele anders nummeriert, bei ihm entsprechen diesen 19a-c.

Bei Eisenberg entsprechen diesen die unter (5-7) angeführten Beispiele.
} 
Neuauflagen seiner Grammatik im Wortlaut übernommen hat. Vgl. dazu Eisenberg (2013a: 268): „Wir befinden uns hier in einem Übergangsbereich von Wortund Satzgrammatik, der in seiner Systematik noch keineswegs verstanden ist.“

Im Zusammenhang mit dem produktiven Muster, Adjektive als Objektsprädikativ zu transitiven Verben zu verwenden, entdeckt Eisenberg (2013a: 336) strukturelle Parallelen zwischen Adjektiven und Verbpartikeln, was er als Inkorporation von Adjektiven interpretiert und durch die prinzipielle Ersetzbarkeit von Adjektiven durch Partikeln illustriert: „Mit dem Adjektiv wird über das vom direkten Objekt Bezeichnete prädiziert und es hat dabei dieselbe Stellung wie eine Verbpartikel“ (vgl. Eisenberg 2013a: 336). Was die Interpretation der Beispiele in $(5-7)^{6}$ anbetrifft, äußert sich Eisenberg (2013b: 225f.) darüber wie folgt:

Diese Sätze sind wahr, wenn Karl dabei ist, Gabis Fahrrad zu streichen bzw. die Kartoffeln weich zu kochen. Bei inkrementellen Prädikaten kommt es nicht auf das Resultat, sondern auf das Ablaufen des Vorgangs selbst an. Das Adjektiv charakterisiert den Vorgang, es geht um das »Grünstreichen« und um das »Weichkochen«, deren Resultat die Zuschreibung der entsprechenden Eigenschaft sein kann, aber nicht sein muss. (Eisenberg 2013b: 225f.)

Das o.a. Zitat enthält implizit die Annahme, dass der Prozess des Grünstreichens und des Weichkochens im Deutschen als eine konzeptuelle Einheit durch Wortbildungskonstruktionen kodiert wird, deren genauer Status sich jedoch nicht in jedem Fall exakt feststellen lässt, zumal diese ja einerseits zwischen Okkasionalität und Lexikalisiertheit und andererseits zwischen Komposition und Partikelverbbildung oszillieren können. Das nächste Zitat aus Eisenberg (2013b) vergegenwärtigt ebendiese beiden Typen von Übergangszonen.

Die Tendenz des Objektprädikativs zur Inkorporierung ins Verb führt dazu, dass der adjektivische Bestandteil im Verhalten einer Verbpartikel nahe kommt (krankschreiben, gesundbeten, kaputtmachen wie abgeben, aufhängen, weglaufen, 20; Wort, 8.4). (Eisenberg 2013b: 233)

${ }_{6} \quad$ Diese sind bei ihm als Bespiele (17) a., b. und c. wiedergegeben. 
Festzuhalten ist auf jeden Fall, dass der grammatische Status der einzelnen Komponenten der Resultativkonstruktionen im Deutschen und Ungarischen jeweils unterschiedlich ist. Die scheinbar adjektivischen Konstruktionsglieder entpuppen sich bei näherem Hinsehen im Deutschen entweder als Kompositionsglieder oder als erste Konstituenten von Partikelverben, und im Ungarischen als durch eine direktionale adverbiale Kasusendung markierte deadjektivische Nomina, die entweder als elliptische Nominalphrasen oder als Konversionen von Adjektiven zu deuten sind.

\section{Bildschemata im Sprachvergleich Deutsch-Ungarisch}

Im Folgenden soll die kontrastive Porträtierung der deutschen und ungarischen Resultativkonstruktionen aus der Perspektive der Bildschemata vorgenommen werden. Bei der Definition der Bildschemata (,image schemas') stütze ich mich auf Johnson (1987), der im Rahmen seiner holistischen kognitiven Semantik dem Aspekt der Körperbezogenheit eine wichtige Rolle zuschreibt und sensomotorische Erfahrungen als unhintergehbar ansieht. Bildschemata entstehen demnach aus elementaren vorbegrifflichen Körpererfahrungen als einfache Muster von Wahrnehmungen und Bewegungsabläufen. Sie sind weder konkrete Bilder noch abstrakte Propositionen, sondern eher holistische Gestalten mit einfachen internen Strukturen, die auf unendlich viele Wahrnehmungen und Ereignisse Bezug nehmen können (vgl. Johnson 1987: 22ff.). Sie gelten als Grundform kognitiver Modelle, bieten eine Basis für die körperbezogene Kognition und stellen somit eine Vermittlungsinstanz zwischen Subjekt und Welt dar. Es ist mit Schröder zu betonen, dass Bildschemata „keine mentalen Vorstellungsbilder, sondern allgemeine Grundmuster der perzeptuellen und motorischen Interaktion des Menschen mit seiner Umwelt“ sind (vgl. Schröder 2012: 35).

Im Folgenden werde ich mich auf die kontrastive Analyse derjenigen Bildschemata konzentrieren, die die Resultativkonstruktionen im Deutschen und Ungarischen motivieren. Johnson (1987: 126) sind u.a. folgende Beispiele für Bildschemata zu entnehmen: BehäLter (CONTAINer), PFAD (PATH), QUelle (SOURCE)-PFAD (PATH)-ZIEL (GOAL), OBERFLÄCHE (SURFACE), ZENTRUM-PERIPHERIE (CENTER-PERIPHERY), TEIL-GANZES (PART-WHOLE) ODER VOLL-LEER (FULL-EMPTY). 
Für die Belange der vorliegenden Analyse sind vor allem die Bildschemata BEHÄLTER, PFAD und OBERFLÄCHE bZW. QUELLE-PFAD-ZIEL unumgänglich. Da Resultativkonstruktionen nicht einfach konkrete räumliche Verhältnisse widerspiegeln, sondern auch das abstrakte Konzept RESUlTAT voraussetzen, gehe ich davon aus, dass hier im Zuge der metaphorischen Bedeutungskonstruierung Bildschemata auf einen abstrakten Erfahrungsbereich projiziert werden, wobei die metaphorischen Modelle HANDLUNG IST BEWEGUNG EINES OBJEKTS IM RAUM sowie erfolgreich handeln ist zurücklegung eines pfades bzw. ERFOLG IST ERREICHEN EINES ZIELES aktiviert werden. In meiner Analyse werde ich auf die den Resultativkonstruktionen zugrunde liegenden Bildschemata fokussieren und die o.a. metaphorischen Projektionen werde ich nicht jedes Mal erwähnen, zumal sie transparent und leicht nachvollziehbar sind und auf eine standardisierte Art und Weise vonstattengehen.

Die auffälligsten interlingualen Kontraste zeichnen sich bei der vergleichenden Betrachtung der adjektivischen Resultativkonstruktionen im Deutschen und ihrer ungarischen Entsprechungen ab. Als erstes ist hier festzuhalten, dass die adjektivischen Resultativkonstruktionen im Deutschen überhaupt nicht durch Bildschemata strukturiert sind. Im Folgenden sollen einige einschlägige Beispiele angeführt werden:

(8) $10 \mathrm{~g}$ Butter in einer Pfanne erhitzen, Brot zufügen und bei schwacher bis mittlerer Hitze unter Wenden goldgelb rösten, herausnehmen. Dill waschen, trocken schütteln und fein hacken. https://www.lecker.de/ ostfriesische-fischsuppe-4579.html [gesehen am 03.01.2019]

(9) Die Putenstücke sollen für dieses Rezept möglichst dünn sein. Dazu werden sie in Klarsichtfolie gepackt und dann vorichtig auf Milimeterdicke flach geklopft. WDR, Hrsg, (2006). Familienrezepte 2. Noch mehr leckere Rezepte der WDR Zuschauer. Köln: Schlütersche Verlagsgesellschaft, S. 16.

(10) Dieser Mehrwert kann darin bestehen, dass Teile in einer Fabrik montiert werden, dass ein Buch geschrieben wird, dass Patienten gesund gepflegt werden, usw. (North, Klaus / Friedrich, Peter / Bernhard, Maja (2009). Die Gesundheitshebel: Partizipative Gesundheitsförderung in der Pflege. Wiesbaden: Gabler Verlag, S. 49.) 
Für alle im Deutschen belegten adjektivischen Resultativkonstruktionen ist ausnahmslos festzuhalten, dass Adjektiv und Verb jeweils ohne die Zuhilfenahme von freien oder gebundenen grammatischen Morphemen eine komplexe konzeptuelle Einheit bilden, die nicht über die Wortstrukturgrenzen hinausgeht und die somit den Kriterien einer Wortbildungskonstruktion gerecht wird. ${ }^{7}$ Die Gesamtbedeutung lässt sich aufgrund der lexikalischen Bedeutung des Adjektivs und des Verbs sowie aufgrund der Interdependenz der beiden innerhalb der Wortbildungskonstruktion unter Einbeziehung weiterer kontextueller und situativer Informationen ${ }^{8}$ ermitteln. Die Beteiligung von Bildschemata an der Bedeutungskonstruierung lässt sich dabei weder konstruktionsextern noch -intern nachweisen. Die metaphorische Projektion erfolgt ohne die Dazwischenschaltung von Bildschemata, unmittelbar unter Einbeziehung der metaphorischen Modelle HANDLUNG IST BEWEGUNG EINES OBJEKTS IM RAUM SOWI ERFOLG IST ERREICHEN EINES ZIELES.

Im Ungarischen lässt sich in scharfem Kontrast zur Faktenlage im Deutschen bei allen Resultativkonstruktionen - d.h. sowohl bei den Äquivalenten der deutschen adjektivischen als auch der deutschen direktionalen Konstruktionen - die maßgebende Bedeutung von Bildschemata beobachten. Festzuhalten ist außerdem die erstaunliche Einheitlichkeit der die ungarischen Resultativkonstruktionen strukturierenden Bildschemata, zumal die ungarischen Entsprechungen aller im Deutschen belegten Konstruktionstypen mit Ausnahme von Konstruktionen mit der direktionalen PP $z u+$ Dativ einheitlich durch das Bildschema kontaktaufnahme mit einer oberfläche motiviert sind, wobei alle einschlägigen Exemplare am Objektsprädikativ einheitlich die Kasusendung -ra/-re aufweisen. Kothencz (2007: 441) visualisiert die durch die adverbialen Kasusendungen kodierten räumlichen Verhältnisse in einer übersichtlichen Abbildung, die hier adaptiert und ins Deutsche übersetzt ${ }^{9}$ als Abbildung 1 wiedergegeben ist.

\footnotetext{
Die Problematik der jeweils gültigen Rechtschreibregeln kann hier aus Platzgründen nicht angeschnitten werden.

8 Der zur Bedeutungserschließung nötige Anteil an kontextuellen und situativen Informationen hängt entscheidend von dem Lexikalisiertheitsgrad der jeweiligen Wortbildungskonstuktion ab.

9 Die Übersetzungen stammen von der Autorin dieses Aufsatzes.
} 
-ra/-re NÄHERKOMMEN SUBLATIV

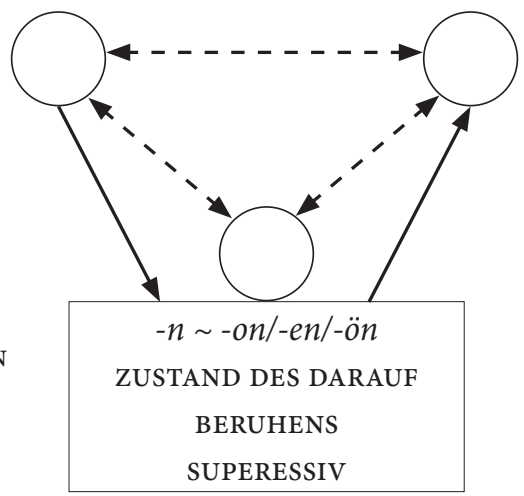

-ról/-ről

DELATIV

Abb. 1: Die Kasusendungen -ra/-re, -n -on/-en/-ön und -ról/-röl in ihrem Verhältnis zueinander

Für unsere Belange ist die Visualisierung der Endung -ra/-re als morphologische Markierung für die Kodierung des Kasus Sublativ von besonderer Relevanz. Der Abbildung lässt sich entnehmen, dass hier das Bildschema NÄHERKOMMEN $^{10}$ (AN EINE OBERFLÄCHE) aktiviert wird.

Bei den Entsprechungen der deutschen Resultativkonstruktionen wird im Ungarischen durch die Beteiligung von -ra/-re-Konstruktionen das Bildschema KontaKtaufnahme Mit eINer Oberfläche aktiviert. Die genaue Wortartzugehörigkeit der Objektsprädikative ist allerdings umstritten, zumal sich die Kasusendung -ra/-re gewöhnlich nur mit Substantiven verbinden lässt, sodass entweder eine elliptische attributive Konstruktion oder die Konversion des Adjektivs zu einem Substantiv für die Analyse in Betracht zu ziehen ist. ${ }^{11}$ Einfachheitshalber werde ich diese als deadjektivische Konstruktionen bezeichnen.

Im Folgenden sollen die deadjektivischen -ra/-re-Resultativkonstruktionen durch Bespiele illustriert werden.

\footnotetext{
10 Kothencz (2007: 441) spricht hier einfach von NÄHerkommen, ich plädiere jedoch für die Präzisierung des einschlägigen Bildschemas und schlage dafür KONTAKTAUfNAHME MIT EINER OBERFLÄCHE vor.

11 Welche dieser Analysealternativen zu bevorzugen wäre, kann hier aus Umfangsgründen nicht andiskutiert werden.
} 
(11) A fejet, a farkat és az apróra vágott hagymát egy lábasba tesszük, annyi vizet adunk hozzá, hogy az alapanyagokat ellepje. Majd kb. 45 perc alatt puhára fözzük. https://www.nosalty.hu/receptek/kategoria/levesek/ halaszle [gesehen am 01.03.2019]

(12) A bekevert masszát papírral fedett sütőlemezre adagoljuk, egyenletesen 10-15mm vastagságúra kenjük, és $160^{\circ} \mathrm{C}$-on sütjük.

kepzesevolucioja.hu/dmdocuments/4ap/18_0536_016_101215.pdf [gesehen am 15.03.2019]

(13) A cipókat egyenként ujjnyi vastagra nyújtjuk, majd megkenjük az olvasztott vajjal. www.dkvk.hu/feltoltkepek/szulinapi_receptek.pdf [gesehen am 15.03.2019]

Im Ungarischen sind jedoch nicht nur deadjektivische -ra/-re-Konstruktionen weit verbreitet, sondern auch substantivische, die ebenfalls sehr produktiv sind. Im Folgenden sollen letztere auch durch illustrative Beispiele vorgeführt werden.

(14) A 2000-es években nagy divat volt csíkra szedetni a szemöldököt, [...] https://www.glamour.hu/.../9-sztar-aki-teljesen-megvaltozott-aszemoldokenek-formaja-miatt [gesehen am 22.01.2019]

(15) A vöröshagymákat tisztítsuk meg, és vágjuk apró kockákra. https://www. nosalty.hu/receptek/kategoria/levesek/halaszle [gesehen am 01.03.2019]

(16) A pontyfiléket hajszálvékony csíkokban beirdaljuk, majd ujjnyi vastag csíkokra vágjuk, és besózzuk.

https://www.nosalty.hu/receptek/kategoria/levesek/halaszle [gesehen am 01.03.2019]

Aufgrund der Analyse der unter (11-16) angeführten Beispiele ist im Hinblick auf die Faktenlage im Ungarischen festzuhalten, dass sich hier alle Subtypen der resultativen -ra/-re-Konstruktionen einheitlich verhalten und alle ausnahmslos durch das Bildschema KONTAKTAUfNAHME MIT EINER OBERFLÄCHE motiviert sind. Die Beteiligung dieses Bildschemas an der metaphorischen Konstruierung der Gesamtbedeutung der resultativen Konstruktionen lässt sich dabei insgesamt sowohl konstruktionsextern als auch -intern nachweisen. 
Die metaphorische Projektion erfolgt mit Hilfe der Dazwischenschaltung des einschlägigen Bildschemas, unter Einbeziehung der metaphorischen Modelle HANDLUNG IST BEWEGUNG EINES OBJEKTS IM RAUM SOWIe ERFOLG IST ERREICHEN EINES ZIELES. In Abbildung 2 wird die Beteiligung des Bildschemas KONTAKTAUFNAHME MIT EINER OBERFLÄCHE an der Aktivierung der resultativen -ral-re-Konstruktion visualisiert.

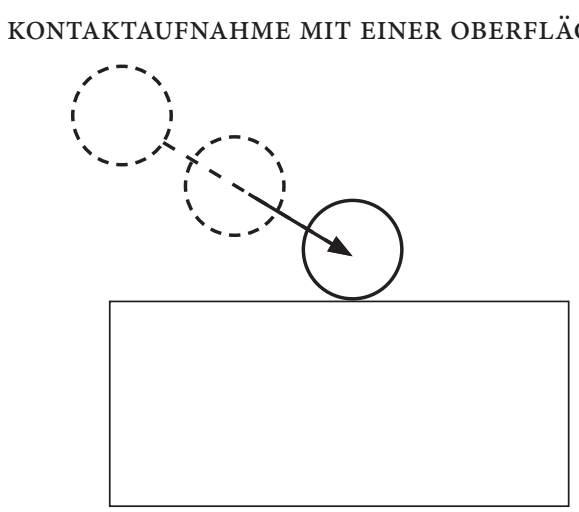

Abb. 2: Bildschema bei -ra/-re-Konstruktionen

Die deutschen Entsprechungen der prototypischen substantivischen $r a /$-re-Konstruktionen sind ausnahmslos in + Akk-Konstruktionen, die einheitlich durch das Bildschema INS INNERE EINES BEHÄLTERS GELANGEN motiviert sind. Vgl. dazu die Visualisierung in Abbildung 3.

INS INNERE EINES BEHÄLTERS GELANGEN

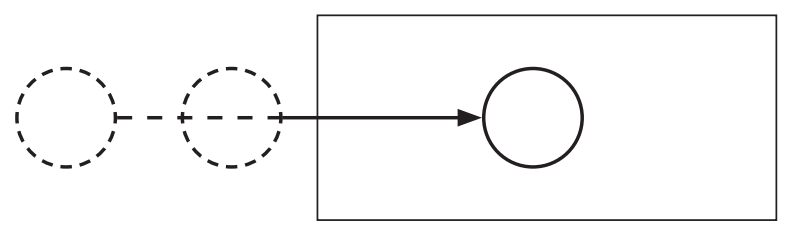

Abb. 3: Bildschema bei in + Akkusativ-Konstruktionen 
Die in + Akkusativ-Konstruktion sei im Folgenden durch einige Beispiele veranschaulicht.

(17) Fisch filetieren, in große Würfel schneiden und die Gräten aufbewahren. https://www.lecker.de/fischsuppe-23527.html [gesehen am 03.01.2019]

(18) Zwiebeln schälen und in Scheiben schneiden. Paprika putzen, waschen und in Stücke schneiden. https://www.lecker.de/fischsuppe-23527.html [gesehen am 03.01.2019]

(19) Dabei ging man zunächst nach der Methode zu Werke, daß man Brecht fein säuberlich in zwei Hälften tranchierte: in einen großen Dichter und in einen weniger großen Politiker und Parteigänger. (Der Spiegel 32, (1978), S. 217)

Die deutschen $z u+$ Dativ-Konstruktionen finden schließlich ihre Entsprechungen in den ungarischen -vá/-vé-Konstruktionen. Die Funktion dieser Konstruktionen besteht in der Perspektivierung der vollständigen Zustandsveränderung, wobei nicht einfach nur Form, Gestalt, Konsistenz oder Farbe einer Entität eine Veränderung erfährt, sondern die Entität als Ganzheit als eine andere Entität perspektiviert wird. Alle Exemplare der deutschen $z u+$ Dativ-Konstruktionen sind einheitlich durch das Bildschema IN DIE NÄHE DES ZIELS GELANGEN motiviert. Vgl. dazu Abbildung 4 zur visuellen Vergegenwärtigung dieses Bildschemas.

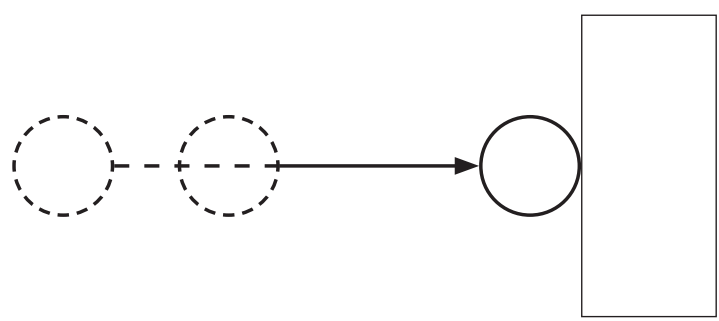

Abb. 4: Bildschema bei $z u+$ Dativ-Konstruktionen

Die folgenden Beispiele sollen die Produktivität dieser Konstruktionen im Deutschen (vgl. die Belege unter 20-22) und im Ungarischen (vgl. die Belege unter 23-25) veranschaulichen. 
(20) Schneemasse: Eiweiß, Kristallzucker \& Prise Salz zu geschmeidigem Schnee schlagen, Weizenpuder unterheben. (Rezeptblatt. (2017): Tourismusschulen Bad Ischl, Salzkammergut, S. 1)

(21) Mehl, Kakao und Salz in einer separaten Schüssel vermischen und anschließend zur Buttermasse hinzugeben und alles miteinander zu einem homogenen Teig kneten. https://lerch-genuss.de/blog/in-derweihnachtsbaeckerei-plaetzchenrezepte/ [gesehen am 17.03.2019]

(22) Den Teig zu einer Kugel formen und leicht flach drücken. https://lerch-genuss.de/blog/in-der-weihnachtsbaeckereiplaetzchenrezepte/ [gesehen am]

(23) Az amerikai kormány tudósai kifejlesztettek egy eljárást, melynek segítségével nanoszekundumok alatt jéggé lehet fagyasztani a vizet. https://index.hu/tudomany/fgys070319/ [gesehen am 17.03.2019]

(24) [...] egy olyan világot képzel el magának, ahol nem a masszává olvadt kvarc és a homok, hanem az idő az, amit üveglappá húznak, vagy ballonná és gömbbé fújnak az üveghuták.

http://balkon.art/home/deli-agnes-hullam/ [gesehen am 17.03.2019]

(25) Megveszem a hársfa rönköt. Kifürészeltetem, magam gyalulom, fürészelem léccé.

http://meheszklub.hu/index.php?topic=719.30 [gesehen am 17.03.2019]

Während die $z u+$ Dat-Konstruktionen durch das Bildschema IN DIE NÄHE DES ZIELS GELANGEN motiviert sind, lassen sich bei den -vál-vé-Konstruktionen keinerlei Bildschemata ermitteln, zumal diese in der ungarischen Gegenwartssprache mit der Kodierung räumlicher Relationen nicht mehr in Verbindung zu bringen sind. Im Gegensatz zu Kothencz (2011: 87), die Beispiele wie Péter békából királyfivá változott [Péter verwandelte sich von einem Frosch in einen Prinzen] mit Hilfe des Bildschemas QUELLE-PFAD-ZIEL interpretiert, argumentiere ich dafür, ausschließlich bei der Analyse historischer Daten ein Bildschema anzusetzen. Die Endung -vá/-vé lässt sich nämlich diachron auf das Morphem -á/-é zurückführen, welches in der altungarischen Epoche als Lativ-Endung ${ }^{12}$ gedient hatte (vgl. Korompay 1991, angeführt in Kothencz

12 Der Lativ hatte in der altungarischen Epoche eine konkret räumliche Funktion, und zwar die Kodierung des Ziels einer Bewegung. 
2011: 36). In der ungarischen Gegenwartssprache ist die monoseme -vál-véEndung jedoch eindeutig und ausschließlich mit der Kodierung des adverbialen Kasus Translativ-Faktiv verknüpft und dient der Resultatsbestimmung (vgl. Ladányi 2017: 586). Da im heutigen Ungarisch durch -vál-vé produktiv keine räumliche Bedeutungsrelation ${ }^{13}$ ausgedrückt werden kann, halte ich die Annahme eines Bildschemas in der Analyse der einschlägigen Konstruktion für nicht begründet.

\section{Fazit und Ausblick}

Die Ergebnisse der tentativen kontrastiven Analyse lassen sich in einer tabellarischen Übersicht wie folgt zusammenfassen:

\begin{tabular}{|l|c|c|c|}
\hline \multirow{3}{*}{ Deutsch } & Adj + Verb WBK & $\begin{array}{c}\text { in }+ \text { Akk } \\
\text { Konstruktion }\end{array}$ & $\begin{array}{c}z u+\text { Dat } \\
\text { Konstruktion }\end{array}$ \\
\cline { 2 - 4 } & kein Bildschema & $\begin{array}{c}\text { INS INNERE EINES } \\
\text { BEHÄLTERS GELANGEN }\end{array}$ & $\begin{array}{c}\text { IN DIE NÄHE DES } \\
\text { ZIELS GELANGEN }\end{array}$ \\
\hline Ungarisch & $\begin{array}{c}-r a /-r e- \\
\text { Konstruktion }\end{array}$ & $\begin{array}{c}\text {-vál-vé- } \\
\text { Konstruktion }\end{array}$ \\
\cline { 2 - 4 } & KONTAKTAUFNAHME MIT EINER OBERFL ̈̈CHE & kein Bildschema \\
\hline
\end{tabular}

Tab. 1: Resultativkonstruktionen und Bildschemata im Sprachvergleich Deutsch-Ungarisch

Wie Tabelle $1 \mathrm{zu}$ entnehmen ist, lässt sich als Ergebnis der kontrastiven Analyse insgesamt ein klares und übersichtliches Gesamtbild der interlingualen Zuordnungsmöglichkeiten von Resultativkonstruktionen und Bildschemata in deutsch-ungarischer Relation zeichnen. Die deutschen Adj + Verb-Konstruktionen, die ungeachtet der orthographischen Variation einheitlich als Wortbildungskonstruktionen analysiert werden, und die durch keinerlei Bildschemata motiviert sind, und die in + Akk-Konstruktion, die durch das Bildschema INS INNERE EINES BEHÄLTERS GELANGEN strukturiert ist, haben als gemein-

\footnotetext{
13 In Keszler / Lengyel (2008: 81) wird ein einziges Beispiel für Lokalbestimmung bei -vá/-vé erwähnt: „világgá megy 'er/sie zieht in die Welt los.“ Hier handelt es sich jedoch um einen Archaismus, der als eine isolierte idiomatische Konstruktion anzusehen ist.
} 
same Entsprechung im Ungarischen die -ra/-re-Konstruktion, die durch das Bildschema KontakTAUfNAHME MIT EINER OBERFLÄCHe motiviert ist. Die deutsche $z u+$ Dat-Konstruktion, der das Bildschema IN DIE NÄHE DES ZIELS GELANGEN zugrunde liegt, findet dafür in der durch keinerlei Bildschemata motivierten -vál-vé-Konstruktion ihre ungarische Entsprechung.

Die o.a. Ergebnisse der tentativen kontrastiven Analyse laden einerseits zur theoretischen Vertiefung ein und andererseits zur Anwendung im Fremdsprachenunterricht, in der Lernerlexikographie sowie in Theorie und Praxis der Übersetzung. In theoretischer Hinsicht öffnen sich vor allem im Hinblick auf die Einbeziehung weiterer resultativer Konstruktionstypen sowie weiterer - in typologischer und kultureller Hinsicht möglichst auch weit entfernter - Sprachen neue Forschungsperspektiven. In diesem Sinne versteht sich die vorliegende Arbeit auch als ein erster bescheidener Baustein zur kontrastiven Typologie der Resultativkonstruktionen. Die praxisbezogenen Aspekte zukünftiger Forschung können dafür entweder an die bereits reichen Traditionen der Vermittlung von Bildschemata und Metaphern im DaF-Unterricht ${ }^{14}$ anknüpfen oder experimentelles und methodologisches Neuland betreten.

\section{Literatur}

Ágel, Vilmos (2017): Grammatische Textanalyse: Textglieder, Satzglieder, Wortgruppenglieder. Berlin / Boston: de Gruyter.

Brdar-Szabó, Rita (2019): Rezultatív mikrokonstrukciók és morfológiai vetélytársaik elmélet és empíria egységében. [Resultative Mikrokonstruktionen und ihre morphologischen Konkurrenten in Theorie und Empirie] Vortragsmanuskript für die Tagung „Nyelvtan, diskurzus, megismerés: A magyar nyelv funkcionális leírása.“ 28-29. März 2019, Budapest: Eötvös-Loránd-Universität.

Broccias, Cristiano (2004): The cognitive basis of adjectival and adverbial resultative constructions. In: Annual Review of Cognitive Linguistics 2, 103-126.

14 Vgl. dazu u.a. Roche / Suñer (2014), Kanaplianik (2016), Roche / EL-Bouz (2018) und Roche / Suñer, Hrsg. (2017). 
Broccias, Cristiano (2008): Towards a history of English resultative constructions: the case of adjectival resultative constructions. In: English Language and Linguistics 12, 27-54.

Eisenberg, Peter (1989): Grundriß der deutschen Grammatik. 2., überarbeitete und ergänzte Auflage. Stuttgart: J. B. Metzlersche Verlagsbuchhandlung.

Eisenberg, Peter (1998): Grundriß der deutschen Grammatik. Band 1: Das Wort. Stuttgart / Weimar: Verlag J. B. Metzler.

Eisenberg, Peter (2013a): Grundriss der deutschen Grammatik. Band 1: Das Wort. Unter Mitarbeit von Rolf Thieroff. 4., aktualisierte und überarbeitete Auflage. Stuttgart / Weimar: Verlag J. B. Metzler.

Eisenberg, Peter (2013b): Grundriss der deutschen Grammatik. Band 2: Der Satz. Unter Mitarbeit von Rolf Thieroff. 4., aktualisierte und überarbeitete Auflage. Stuttgart / Weimar: Verlag J. B. Metzler.

Goldberg, Adele (1995): Constructions: A construction grammar approach to argument structure. Chicago: The University of Chicago Press.

Goldberg, Adele, Jackendoff, Ray (2004): The English resultative as a family of constructions. In: Language 80, 532-568.

Helbig, Gerhard (2008): Zu den Objektsprädikaten und ihren einzelnen Klassen. In: Czachur, Waldemar / Czyżewska, Marta (Hrsg.): Vom Wort zum Text. Studien zur deutschen Sprache und Kultur. Festschrift für Professor Józef Wiktorowicz zum 65. Geburtstag. Warszawa: Instityt Germanystiki, 193-213. Johnson, Mark (1987): The Body in the Mind: The Bodily Basis of Meaning, Imagination and Reason. Chicago: University of Chicago Press.

Kanaplianik, Katsiaryna (2016): Kognitionslinguistisch basierte Animationen für die deutschen Modalverben. Berlin: Lit Verlag.

Keszler, Borbála / Lengyel, Klára (2008): Ungarische Grammatik. Aus dem Ungarischen übertragen von einer Übersetzergruppe unter der Leitung von Borbála Keszler und Attila Péteri. Hamburg: Helmut Buske Verlag.

Korompay, Klára (1991): A névszóragozás. [Die Deklination der Nomina.] In: Benkő, Loránd (Hrsg.): A magyar nyelv történeti nyelvtana I. A korai ómagyar kor és előzményei. [Historische Grammatik des Ungarischen I. Die frühe altungarische Zeit und ihre Vorläufer.] Budapest: Akadémiai Kiadó, 284-318. Kothencz, Gabriella (2007): A -ban/-ben és az - $n \sim$-on/-en/-ön jelentésrendszere. [Das Bedeutungssystem von -ban/-ben und $-n \sim-o n /-e n /-o ̈ n$.$] In: Magyar$ Nyelv 103.4, 432-451. 
Kothencz, Gabriella (2011). Kognitív jelentéstörténeti vizsgálatok a magyar nyelv határozóragjai körében. [Kognitive Untersuchungen zur diachronen Semantik im Bereich der adverbialen Kasussuffixe des Ungarischen.] PhD-Dissertation. Budapest: Loránd-Eötvös-Loránd-Universität.

Kugler, Nóra (2019): Konstrukciók a nyelv(tan)tanításban. [Konstruktionen im Sprach(lehre)unterricht.] Manuskript. Budapest: Loránd-Eötvös-Universität. Ladányi, Mária (2017): Alaktan. [Formenlehre.] In: Tolcsvai Nagy, Gábor (Hrsg.): Nyelvtan. [Grammatik.] Budapest: Osiris Kiadó, 503-660.

Levin, Beth (1993): English verb classes and alternations: A preliminary investigation. Chicago: University of Chicago Press.

Roche, Jörg / EL-Bouz, Katsiaryna, unter Mitarbeit von Leuchte, Oliver (2018): Deutsche Grammatik sportlich und animiert. In: Zeitschrift für Interkulturellen Fremdsprachenunterricht 23.1, 30-42.

Roche, Jörg / Suñer Muñoz, Ferran (2014): Kognition und Grammatik. Ein kognitionswissenschaftlicher Ansatz zur Grammatikvermittlung am Beispiel der Grammatikanimationen. In: Zeitschrift für Interkulturellen Fremdsprachenunterricht 19.2, 119-145.

Roche, Jörg / Suñer Muñoz, Ferran (Hrsg.) (2017): Sprachenlernen und Kognition. Grundlagen einer kognitiven Sprachendidaktik. Tübingen: Narr Verlag.

Schröder, Ulrike (2012): Kommunikationstheoretische Fragestellungen in der kognitiven Metaphernforschung: Eine Betrachtung von ihren Anfängen bis zur Gegenwart. Tübingen: Narr Verlag. 